\title{
ALGUNAS REFLEXIONES EN TORNO AL ESTILO DE APRENDIZAJE EMPLEADO POR ESTUDIANTES UNIVERSITARIOS
}

\author{
Marlene Aguirre Chaves ${ }^{1}$
}

\section{Resumen}

Este artículo trata de una investigación realizada a veinte estudiantes universitarios de la carrera de la Enseñanza de Español de la Universidad Nacional.

Su objetivo principal es conocer el estilo de aprendizaje empleado por cada uno de los y las estudiantes, y realizar un análisis de los niveles de conocimientos (cognoscitivo, afectivo-actitudinal y psicomotriz), las actividades utilizadas mediante el uso de verbos de acción y de estado y las técnicas de enseñanza empleadas.

El aprendizaje de los y las estudiantes ha sido abordado por los estudiosos de la educación desde diferentes perspectivas. Algunos de ellos han puesto su mirada en lo interno del aula, destacando aspectos de metodología, de estrategias, de hábitos de estudio, autoestima, fallas en la formación pedagógica de los educadores $\mathrm{y}$, recientemente, se ha puesto énfasis en la forma en que aprenden los estudiantes, es decir, en los estilos de aprendizaje que posee cada persona para asimilar el conocimiento.

Howard Gardner ha tratado el tema de las inteligencias múltiples, que está vinculado con las potencialidades de cada ser humano para aprender. Otros estudiosos de la educación, por el contrario, se han referido a aspectos relacionados con la parte social, política y económica que influyen de manera indirecta, pero enfática, en los procesos de enseñanza y aprendizaje.

Se hace un planteamiento breve desde la perspectiva teórica respaldada en las opiniones de algunos investigadores universitarios y una fundamentación áulica desde la praxis, que está estrechamente relacionada con los principales hallazgos de esta investigación.

Palabras claves: estudiantes universitarios, estilos de aprendizaje.

\section{Abstract}

This article is about an investigation carried out twenty university students of

1 Académica de Educología-CIDE, y durante el 2006 fungió como subdirectora de esa unidad. Licenciada en la Enseñanza del Español. Profesora del Ministerio de Educación Pública, especialista en didáctica del español. 
the career of the Teaching of Spanish of the National University.

The main objective is to know the learning style used by each one of those and the students, and to carry out an analysis of the levels of knowledge (cognitive, affection-attitude and psychometric), the activities used by means of the use of action verbs and of state and the used teaching techniques.

The leaming of those and the students have been approached by the specialists of the education from different perspectives. Some of them, they have put their look to the internal of the classroom, highlighting methodology aspects, of strategies, of study habits, self-esteem, flaws in the pedagogic formation of the educating ones and recently he/she has put on emphasis in the form in that the students leam, concretely, the learning styles that each person possesses to assimilate the knowledge.

Howard Gardner it has treated the topic of the multiple intelligence, one this linked with the potentialities that each human being to leam. Others specialists of the education, on the contrary, they have referred to aspects related with the social part, politics and economic that influences in an indirect, but emphatic way, in the teaching processes and learning.

A brief position is made from the theoretical perspective supported in the opinions of some university investigators and a foundation in the classroom for the practice that is closely related with the main discoveries of this investigation.

Keywords: university students, levels of knowledge.

\section{Una retrospectiva social}

$\mathrm{E}^{1}$ 1 fracaso de las y los estudiantes en sus estudios ha despertado interés entre los y las profesionales de la educación y ha sido tratado de diversas maneras. Recientemente, Rafael Cuevas (2004) ha dado su opinión basada en Los herederos de Pierre Bordieu y Claude Passeron, desde el punto de vista de la sociología, como la disciplina que puede explicar acerca del fracaso escolar.

Cuevas (2004) ha señalado que desde la teoría de Bordieu y Passeron, se plantea un cierto determinismo social, casi una "ley natural", la cual dicta que estudiantes provenientes de clases desfavorecidas no pueden permanecer en la universidad. Según Cuevas, estos autores proponen una línea de trabajo orientada en una pedagogía racional, para contrarrestar la desigualdad cultural que se inicia desde la etapa del kínder hasta la educación superior.

Por otra parte, Gerardo Morales (2004), investigador universitario, manifiesta que el título de Los herederos se debe, efectivamente, que al ingresar a la universidad ya poseen un capital simbólico y cultural heredado de un 
medio socioeconómico que los distingue de muchas maneras: en el uso de la lengua, en el acceso a los bienes culturales, en las posibilidades de conocer otras culturas.

De tal manera, afirma Morales (2004) que la elección está dada mucho antes de su ingreso formal a la universidad y existen condiciones previas "extracurriculares" que distinguen a unos estudiantes de otros, por lo cual aquí la diferencia no es, por supuesto, innata.

Claro está, que en nuestras aulas universitarias se destaca la presencia de estudiantes provenientes de distintas regiones del país; esto hace que existan no sólo desigualdades en torno al aspecto económico, social y político, sino en cuanto a su formación cultural.

El asunto del aprendizaje de los y las estudiantes se torna todavía más complejo, si a lo social, se le une lo económico y las políticas de turno que imperan cada cuatro años en la educación secundaria. Los factores económicos, sociales y políticos son una realidad alejada de la práctica pedagógica. Los y las estudiantes llegan a las aulas universitarias y muchos no pueden superar las deficiencias académicas que el medio o el entorno les ha heredado.

Las deficiencias académicas que se encuentran en el ámbito universitario muestran una sucesión de hechos desde los estudios de la primaria y se acentúan más en la secundaria, cuando el o la estudiante se encuentra en un proceso de definición de su personalidad y requiere de una orientación básica por parte de sus padres, madres y docentes. No obstante, muchas veces no la encuentra y comienza a gestarse un desequilibrio emocional, que influye en su formación académica.

Se une a lo anterior, la falta de recursos económicos, los problemas familiares: padres divorciados, separados, agresivos, de baja escolaridad y, por ende, con ingresos muy bajos; el bajo rendimiento académico y otros factores que influyen en su aprendizaje. Cuando esto sucede, el o la estudiante termina por desertar de las aulas, con el agravante que puede encontrar refugio en las drogas, el alcoholismo y otras prácticas que lo conducen a un deterioro en su formación y calidad de vida.

Sin embargo, el problema no termina ahí; la baja escolaridad incide en la contratación de sus empleos. Así lo demuestra Jorge Nowalski (2003), en su libro Asimetrías económicas, sociales y políticas en Costa Rica, en un estudio de la PNUD, donde se afirma que: "la educación es clave para avanzar hacia un estilo de desarrollo inclusivo al incidir directamente en el curso de vida de las personas desde sus inicios" (p. 267). Además, apunta que la educación tiene un carácter individual y colectivo y es un instrumental para 
lograr una mayor movilidad y cohesión social, transmite valores y principios que rigen y fortalecen a las sociedades (p. 267).

La educación es clave para el desarrollo de la sociedad, lo cierto es que mientras nuestros estudiantes dejan las aulas por las distintas circunstancias que los mediatizan, no existe una política definida por parte de nuestros gobiernos. En cuanto al mejoramiento de programas sociales, queda claramente planteado que se contribuye muy poco con el fortalecimiento de la educación. La pregunta de ¿por qué no aprenden los estudiantes? tendríamos que tratarla, desde luego, haciendo un análisis más riguroso.

La segunda perspectiva está más orientada a señalar los aspectos relacionados con el desarrollo de la praxis en el aula.

\section{La práctica pedagógica en el aula}

Al igual que los problemas sociales, una mala aplicación u orientación de la práctica pedagógica lleva al fracaso de los estudiantes; esto sucede cuando se está clara la forma en que se aborda el aprendizaje y la manera de enseñar de los y las docentes.

En los últimos tiempos, los investigadores de la educación se han dado cuenta que existen grandes deficiencias en la forma en que se evalúan a los estudiantes. Autores como Howard Gardner, con su teoría de las inteligencias múltiples, se ha referido a las distintas habilidades o capacidades que tiene el individuo, las cuales le permiten al ser humano la facilidad de explotar unas áreas más que otras. En un artículo publicado en Internet sobre inteligencias múltiples, de Fernando La Palma (2004), Gardner define el término de inteligencias múltiples como la "capacidad de resolver problemas o elaborar productos que sean valiosos en una o más culturas" (p. 1).

Esta definición amplía el campo de lo que es la inteligencia y dice que la brillantez académica no lo es todo. Según La Palma, hay personas con gran capacidad intelectual, pero incapaces de elegir bien a sus amigos o, por el contrario, hay gente menos brillante en sus estudios, que triunfa en el mundo de los negocios o en su vida personal. Esto quiere decir que triunfa en los negocios, o en los deportes, requiere una inteligencia diferente en cada campo.

El problema está en que a todos los y las estudiantes se les exige poner en práctica habilidades, destrezas y capacidades por igual. Por ejemplo, el 
caso de un estudiante que tiene su habilidad más desarrollada en el área de las matemáticas no así en la de los idiomas o, por el contrario, debe rendir en una prueba en igualdad de condiciones. Esto genera, algunas veces, la desmotivación de los estudiantes, lo que lleva al fracaso escolar y al abandono de las aulas.

Según La Palma (2004), hasta hace poco tiempo, la inteligencia se consideraba como algo innato e inamovible, se nacía inteligente o no; los estudios de Gardner han demostrado lo contrario, al definir la inteligencia como una capacidad, una destreza que se puede desarrollar, sin negar el componente genético. Estas potencialidades las puede desarrollar el ser humano dependiendo del medio ambiente, de sus experiencias y de la educación recibida. Tal afirmación es importante, porque remite a lo que afirmaba Gerardo Morales (2004), que el estudiante al ingresar a la universidad, ya posee una cultura heredada, de un medio socioeconómico y cultural que lo distingue de otros que no la han tenido.

La existencia de información básica de los y las estudiantes, que ingresan por primera vez a la universidad, puede servir de parámetro a cada docente, para conocer más de cerca su condición socioeconómico y las habilidades y destrezas que estos y estas han podido desarrollar mejor en un determinado campo.

Por otra parte, cada estudiante tiene su propio estilo de aprender, así también como cada educador tiene su manera de enseñar. El o la docente aplica técnicas o estrategias didácticas para desarrollar su clase; también sus estudiantes desarrollan sus propias técnicas para aprender.

\section{Referente metodológico}

Para realizar este trabajo, se hizo una encuesta escrita en el 2005, a veinte estudiantes universitarios, que cursan el tercer nivel en la carrera de Bachillerato en la Enseñanza del Español. Básicamente, se les formularon dos preguntas: ¿qué hace usted para aprender? y ¿qué hace la profesora para que usted aprenda? En esta oportunidad, se escogió la primera pregunta, porque la investigadora estaba interesaba en conocer el estilo de aprendizaje, que tenían los y las estudiantes, para adquirir sus conocimientos en la enseñanza de español. 


\section{¿Qué hace usted para aprender?}

$\begin{array}{ll}\text { Estudiante } \mathrm{N}^{\circ} 1 & \text { Para aprender estudio el tema que me inquieta, investigo acerca del tema, lo } \\ \text { repaso constantemente para lograr descifrar aspectos que no he podido enten- }\end{array}$ der.

Como mi manera de aprender es visual, los textos que tengo que leer los suEstudiante $\mathrm{N}^{\circ} 2$ brayo con colores, otras veces, escribo ideas principales y con eso me acuerdo de lo demás.

Estudiante $\mathrm{N}^{\circ} 3$ fuera de clase leo los apuntes, busco información adicional, rescribo y hago esquemas.

Estudiante $\mathrm{N}^{\circ} 4 \quad$ Yo pienso que nadie aprende si no está en actitud de aprendizaje, por lo tanto, cada persona no debe estar dispuesta a aprender cualquier disciplina.

Tener mucha disponibilidad en el aula para poder entender todo. Preguntar

Estudiante $\mathrm{N}^{\circ} 5$ cualquier duda, en cuanto a las lecturas realmente leerlas y sintetizar lo más importante.

Estudiante $\mathrm{N}^{\circ} 6 \quad$ Leer y observar, tengo memoria visual, pero aprendo solo lo que me interesa por lo que procuro interesarme por todo.

Estudiante $\mathrm{N}^{\circ} 7 \quad$ Leer en voz alta e investigar.

Estudiante $\mathrm{N}^{\circ} 8$ Para aprender lo hago por medio de lecturas reflexivas, buscando más información sobre dicho tema para enriquecer el objetivo planteado.

Estudiante $\mathrm{N}^{\circ} 9$

Trato de poner atención en clase, investigo en tiempo libre, entiendo lo que leo y lo pongo en práctica.

Primero, hago una lectura silenciosa y subrayo lo más importante, luego trato de hacer un esquema sólo con los términos claves, para que a la hora de re-

Estudiante $\mathrm{N}^{\circ} 10$ pasar, pueda relacionar los términos con la lectura general y así memorizar para después poder tener un conocimiento total y poder responder a cualquier interrogante o hacer comentarios.

Estudiante $\mathrm{N}^{0} 11 \quad$ Lectura global y resumen o esquematización. Busco material afin que fortalezca lo conocido y busco espacios ordenados y bien iluminados.

Leo el texto asignado, debe haber completo silencio. Lo subrayo (los aspectos

Estudiante $\mathrm{N}^{\circ} 12$ que me parecen más importantes), por último, hago un resumen de lo que subrayé. Lo leo y trato de comprenderlo.

Estudiante $\mathrm{N}^{\circ} 13$ Leo, escucho y me imagino alguna situación que me ha pasado o que me podría pasar para hacer una relación, así sí comprendo y aprendo.

Si es una lectura la analizo detalladamente y trato de comprender hasta el más

Estudiante $\mathrm{N}^{\circ} 14$ pequeño mensaje, utilizo mucho la técnica del mapa conceptual y esquemas, estudio en un lugar aislado donde no reciba mucho sonido esto para una mejor concentración. 

$\begin{array}{ll}\text { Estudiante } \mathrm{N}^{\circ} 15 & \begin{array}{l}\text { Pongo atención en clase, reflexiono acerca de lo expuesto por el profesor y } \\ \text { hago un esfuerzo para comprender las lecturas asignadas. }\end{array}\end{array}$

Estudiante $\mathrm{N}^{\circ} 16$ Aprendo leyendo y aplicando lo que leo, además, trato de explicarle a las demás personas ejemplificando y comentando con los compañeros. $\begin{array}{ll}\text { Estudiante } N^{\circ} 17 & \begin{array}{l}\text { Lectura de materia. Relacionar tipo de mapa conceptual. Resumen mental de } \\ \text { puntos modulares. }\end{array}\end{array}$

Yo aprendo de manera visual y auditiva, en realidad, hago una mezcla de los dos sentidos. A nivel universitario, pongo atención al mensaje que el profesor

Estudiante $\mathrm{N}^{\circ} 18$ está dando, después cuando llega el momento de analizar, estudiar y memorizar la información necesito casi obligatoriamente escribir la materia porque es una forma de que se me grabe permanentemente.

Estudio cuando estoy sola en mi casa. Me gusta estudiar en la noche, a veces escucho música instrumental. Para aprender sobre un tema específico necesito Estudiante $\mathrm{N}^{\circ} 19$ total concentración, para iniciar una lectura previa, posteriormente voy resaltando con un marcador los elementos más relevantes, luego los asocio con algo representativo para mí; este método me permite interiorizar la materia.

Estudiante $\mathrm{N}^{\circ} 20$

Primero que todo analizar. Tratar siempre de hacer relaciones. Hacer lo posible para que lo que estoy aprendiendo pueda relacionarlo con cosas de la realidad y mi realidad; pues los temas abstractos son indescifrables para mí.

Fuente: Consulta realizada a estudiantes universitarios en el curso de Desafios Didácticos (2005).

Para interpretar la información, se procedió a leer detenidamente cada uno de los pasos que siguió el alumno para aprender, los cuales están representados por verbos de acción y proceso (véase la matriz $\mathrm{N}^{\circ} 2$ ).

\begin{tabular}{|c|c|}
\hline \multicolumn{2}{|c|}{$\begin{array}{c}\text { ¿Cómo aprenden los estudiantes universitarios consultados? } \\
\text { Matriz } \mathrm{N}^{\circ} \text { ? }\end{array}$} \\
\hline Estudiante $\mathrm{N}^{\circ} 1$ & $\begin{array}{l}\text { Secuencia de los procesos de aprendizaje } \\
\\
\text { Estudio el tema. } \\
\text { Investigo. } \\
>\text { Repaso. } \\
>\text { Descifro. }\end{array}$ \\
\hline Estudiante $\mathrm{N}^{\circ} 2$ & $\begin{array}{l}\text { Aprendo visual. } \\
\text { Subrayo con colores el texto. } \\
>\text { Escribo ideas principales. } \\
>\text { Recuerdo. }\end{array}$ \\
\hline Estudiante $\mathrm{N}^{\circ} 3$ & $\begin{array}{l}\text { Me concentro en clase. } \\
\text { Leo los apuntes. } \\
\text { Busco información adicional. } \\
>\text { Hago esquemas. }\end{array}$ \\
\hline
\end{tabular}




\begin{tabular}{|c|c|}
\hline Estudiante $\mathrm{N}^{\circ} 4$ & $\begin{array}{l}\text { Para aprender me pongo en actitud de aprendizaje. } \\
\text { Cada persona no aprende cualquier disciplina. }\end{array}$ \\
\hline Estudiante $\mathrm{N}^{\circ} 5$ & $\begin{array}{l}\text { Disponibilidad para entender todo. } \\
\text { Pregunto. } \\
\text { Leo las lecturas. } \\
\text { Sintetizo lo más importante. }\end{array}$ \\
\hline Estudiante $\mathrm{N}^{\circ} 6$ & $\begin{array}{l}>\text { Leo. } \\
>\text { Observo. } \\
>\text { Memorizo en forma visual. } \\
>\text { intereso por todo. }\end{array}$ \\
\hline Estudiante $\mathrm{N}^{\circ} 7$ & $\begin{array}{l}\text { Leo en voz alta. } \\
\text { Investigo. }\end{array}$ \\
\hline Estudiante $\mathrm{N}^{\circ} 8$ & $\begin{array}{l}>\text { Hago lecturas reflexivas. } \\
\text { Busco información adicional. } \\
\text { Enriquezco el objetivo planteado. }\end{array}$ \\
\hline Estudiante $\mathrm{N}^{\circ} 9$ & $\begin{array}{l}>\text { Pongo atención en clases. } \\
>\text { Investigo. } \\
>\text { Trato de entender lo que leo. } \\
>\text { Lo pongo en práctica. }\end{array}$ \\
\hline Estudiante $\mathrm{N}^{\circ} 10$ & $\begin{array}{l}\text { Leo en forma silenciosa. } \\
\text { Subrayo lo más importante. } \\
\text { Hago esquemas con términos claves. } \\
\text { Repaso. } \\
\text { Relaciono los términos con lectura general. } \\
\text { Memorizo para responder a cualquier pregunta o comentario. }\end{array}$ \\
\hline Estudiante $\mathrm{N}^{\circ} 11$ & $\begin{array}{l}>\text { Hago una lectura global. } \\
>\text { Elaboro resumen o esquema. } \\
>\text { Busco material afin. } \\
>\text { Busco espacios ordenados e iluminados. }\end{array}$ \\
\hline Estudiante $\mathrm{N}^{\circ} 12$ & $\begin{array}{l}\text { Leo de manera general. } \\
\text { Debe haber completo silencio. } \\
\text { Subrayo aspectos importantes. } \\
\text { Hago un resumen de lo subrayado. } \\
>\text { Leo el resumen y trato de entenderlo. }\end{array}$ \\
\hline Estudiante $\mathrm{N}^{\circ} 13$ & $\begin{array}{l}\text { Leo. } \\
>\text { Escucho. } \\
>\text { Imagino. } \\
>\text { Relaciono. } \\
>\text { Aprendo. }\end{array}$ \\
\hline Estudiante $\mathrm{N}^{\circ} 14$ & $\begin{array}{l}>\text { Analizo detalladamente. } \\
>\text { Trato de comprender. } \\
>\text { Utilizo técnica del mapa conceptual. } \\
>\text { Elaboro esquemas. } \\
>\text { Estudio en un lugar aislado. } \\
>\text { Requiero concentración. }\end{array}$ \\
\hline
\end{tabular}




\begin{tabular}{|c|c|}
\hline Estudiante $\mathrm{N}^{\circ} 15$ & $\begin{array}{l}>\text { Pongo atención. } \\
>\text { Reflexiono acerca de lo expuesto. } \\
>\text { Hago esfuerzo por comprender. }\end{array}$ \\
\hline Estudiante $\mathrm{N}^{\circ} 16$ & $\begin{array}{l}>\text { Aprendo leyendo. } \\
\text { Aplico lo que leo. } \\
\text { Trato de explicar ejemplificando. }\end{array}$ \\
\hline Estudiante $\mathrm{N}^{\circ} 17$ & $\begin{array}{l}\text { Leo la materia. } \\
\text { La relaciono. } \\
\text { Elaboro mapa conceptual. } \\
\text { Resumo mentalmente los puntos modulares. }\end{array}$ \\
\hline Estudiante $\mathrm{N}^{\circ} 18$ & $\begin{array}{l}\text { Aprendo de manera visual y auditiva. } \\
\text { Hago mezcla de los dos sentidos. } \\
\text { Pongo atención. } \\
>\text { Analizo, estudio y memorizo la información. } \\
\text { Escribo la materia para que se me grabe permanente. }\end{array}$ \\
\hline Estudiante $\mathrm{N}^{\circ} 19$ & $\begin{array}{l}\text { Estudio sola en mi casa. } \\
\text { Estudio en la noche. } \\
\text { Escucho música instrumental. } \\
\text { Necesito total concentración. } \\
\text { Resalto con un marcador los elementos más relevantes. } \\
>\text { Luego los asocio con algo representativo para mí. } \\
>\text { Este método me permite interiorizar la materia. }\end{array}$ \\
\hline Estudiante $\mathrm{N}^{\circ} 20$ & $\begin{array}{l}\text { Analizo. } \\
\text { Relaciono las cosas con la realidad y mi realidad. } \\
\text { Los temas abstractos son indescifrables para mí. }\end{array}$ \\
\hline
\end{tabular}

La matriz anterior muestra datos relacionados con el estilo de aprendizaje que tiene cada estudiante, las actividades y técnicas de aprendizaje que realiza para asimilar la materia, las diferentes capacidades que poseen y también el entorno en que se encuentra cada uno de ellos.

Esta experiencia sobre los estilos de aprendizaje está muy relacionada con un estudio de la Universidad de Illinois, donde un grupo de especialistas en educación se interesaron por investigar cómo se podría ayudar a las y los estudiantes a triunfar en la escuela.

Estos investigadores utilizaron varios aspectos para efectuar dicho estudio, entre ellos, los padres y madres de familia, el amor como un valor importante en el aprendizaje, la tensión que se produce por aspectos del aprendizaje, hábitos de estudio, la toma de exámenes, las calificaciones o notas y los recursos. Uno de los primeros factores que se consideró fue el de los estilos de aprendizaje.

Según estos estudiosos, el aprendizaje se refleja en la forma en que el ser humano responde al ambiente que pertenece, a los estímulos sociales, 
emocionales y físicos. Estos investigadores definen el estilo de aprendizaje como la forma en que la información es procesada. Según ellos, no existe estilo de aprendizaje incorrecto. En este caso, la mayoría de estudiantes mostró preferencia por los siguientes estilos de aprendizaje: visual, auditivo o manipulador. Es común, dicen los autores, que se produzca una combinación de estilos de aprendizajes primarios y secundarios.

Para estos autores, los visuales aprenden, mirando la televisión. Cuando tratan de recordar, ven imágenes del pasado, dibujan las cosas en su mente; según su opinión, el $42 \%$ de los y las estudiantes de secundaria caen en esta categoría. Estos tienen problemas cuando están leyendo, porque no visualizan las cosas muy bien y, por lo general, aprenden escuchando y recuerdan los hechos cuando estos son presentados en forma de poemas, cantos o melodías.

Por otra parte, los que aprenden manipulando cosas, les gusta descubrir cómo funcionan los objetos y muchos son exitosos en artes prácticas como carpintería o diseño. Este grupo representa el 50\% de los estudiantes de secundaria y tienen dificultades en contextos tradicionales; así también, sólo el $10 \%$ de los alumnos aprenden bien en forma auditiva; pero el $80 \%$ del proceso de enseñanza se efectúa auditivamente.

Estos estudiosos afirman que una persona puede descubrir que usa diferentes estilos de aprendizaje dependiendo de la situación. En caso de que un niño tenga problemas en la escuela, tendría que investigarse la forma en que el material está siendo presentado. Otro factor importante para aprender es el ambiente. Según ellos, la tradición dice que se debe tener un cuarto sin ruido, bien iluminado y una silla con respaldo vertical, pero algunos estudiantes aprenden mejor en ambientes desordenados, con música a alto volumen, acostados en la cama y con un cuarto no tan bien iluminado.

Cuando se experimentan varios métodos y estilos de aprendizaje, se puede ayudar a los jóvenes a que no se sientan frustrados y mejoren el rendimiento académico.

En el caso de los veinte estudiantes universitarios consultados, se hizo un análisis detallado de los estilos de aprendizaje que utilizan para aprender, de las técnicas más frecuentes y de los niveles de objetivos de los distintos dominios que poseen: cognoscitivo, psicomotriz y afectivo (véase al respecto la matriz $\mathrm{N}^{\circ} 3$ ). 
Interpretación de datos: Cómo aprenden los estudiantes Iatriz $\mathrm{N}^{\prime \prime} 3$

\begin{tabular}{|c|c|c|c|c|c|c|}
\hline & $\begin{array}{l}\text { Nivel cog- } \\
\text { noscitivo }\end{array}$ & $\begin{array}{l}\text { Nivel } \\
\text { afectivo- } \\
\text { actitudinal }\end{array}$ & $\begin{array}{l}\text { Nivel psi- } \\
\text { comotriz }\end{array}$ & $\begin{array}{l}\text { Activida- } \\
\text { des }\end{array}$ & Técnicas & Estilos \\
\hline Estudiante $\mathrm{N}^{\circ} 1$ & Descifrar & $\begin{array}{l}\text { Buscar in- } \\
\text { formación } \\
\text { adicional }\end{array}$ & & $\begin{array}{l}\text { Investigar. } \\
\text { Repasar }\end{array}$ & $\begin{array}{l}\text { Estudia el } \\
\text { tema }\end{array}$ & Cuestionar \\
\hline Estudiante $\mathrm{N}^{\circ} 2$ & $\begin{array}{l}\text { Recuerdo o } \\
\text { memoria }\end{array}$ & & $\begin{array}{l}\text { Trazar } \\
\text { linea y } \\
\text { letras }\end{array}$ & $\begin{array}{l}\text { Subrayar. } \\
\text { Escribir }\end{array}$ & $\begin{array}{l}\text { Subraya } \\
\text { con colo- } \\
\text { res. } \\
\text { Escribe } \\
\text { ideas } \\
\text { principales }\end{array}$ & $\begin{array}{l}\text { Aprendiza- } \\
\text { je visual }\end{array}$ \\
\hline Estudiante $\mathrm{N}^{\circ} 3$ & Recordar & $\begin{array}{l}\text { Busco in- } \\
\text { formación } \\
\text { adicional }\end{array}$ & $\begin{array}{l}\text { Trazar } \\
\text { lineas y } \\
\text { letras }\end{array}$ & Leer & $\begin{array}{l}\text { Leo apun- } \\
\text { tes. } \\
\text { Hago } \\
\text { esquemas }\end{array}$ & $\begin{array}{l}\text { Concentra- } \\
\text { ción }\end{array}$ \\
\hline Estudiante $\mathrm{N}^{\circ} 4$ & & $\begin{array}{l}\text { Me pongo } \\
\text { en actitud } \\
\text { de aprendi- } \\
\text { zaje }\end{array}$ & & & & $\begin{array}{l}\text { No todas } \\
\text { las perso- } \\
\text { nas pueden } \\
\text { aprender } \\
\text { cualquier } \\
\text { disciplina }\end{array}$ \\
\hline Estudiante $\mathrm{N}^{\circ} 5$ & Sintetizar & $\begin{array}{l}\text { Disponibi- } \\
\text { lidad para } \\
\text { entender } \\
\text { todo }\end{array}$ & & $\begin{array}{l}\text { Leer. } \\
\text { Preguntar }\end{array}$ & & Cuestionar \\
\hline Estudiante $\mathrm{N}^{\circ} 6$ & Memoria & $\begin{array}{l}\text { Me intere- } \\
\text { so por todo }\end{array}$ & & Leo & Observo & Visual \\
\hline Estudiante $\mathrm{N}^{\circ} 7$ & & Investigo & & Leer & $\begin{array}{l}\text { Leo en voz } \\
\text { alta }\end{array}$ & Auditiva \\
\hline Estudiante $\mathrm{N}^{\circ} 8$ & $\begin{array}{l}\text { Compren- } \\
\text { der }\end{array}$ & $\begin{array}{l}\text { Busco in- } \\
\text { formación } \\
\text { adicional. } \\
\text { Enriquezco } \\
\text { el objetivo } \\
\text { planteado }\end{array}$ & & Leer & $\begin{array}{l}\text { Lecturas } \\
\text { reflexivas }\end{array}$ & Reflexionar \\
\hline Estudiante $\mathrm{N}^{\circ} 9$ & $\begin{array}{l}\text { Compren- } \\
\text { der }\end{array}$ & $\begin{array}{l}\text { Trato de } \\
\text { entender lo } \\
\text { que leo }\end{array}$ & & Investigar & $\begin{array}{l}\text { Pongo } \\
\text { atención en } \\
\text { clase. } \\
\text { Lo pongo } \\
\text { en práctica }\end{array}$ & $\begin{array}{l}\text { Concentra- } \\
\text { ción }\end{array}$ \\
\hline
\end{tabular}




\begin{tabular}{|c|c|c|c|c|c|c|}
\hline Estudiante $\mathrm{N}^{\circ} 10$ & $\begin{array}{l}\text { Relacionar. } \\
\text { Memorizar }\end{array}$ & & & $\begin{array}{l}\text { Leer. } \\
\text { Repaso }\end{array}$ & $\begin{array}{l}\text { Subrayo lo } \\
\text { más impor- } \\
\text { tante. } \\
\text { Hago } \\
\text { esquemas } \\
\text { con térmi- } \\
\text { nos claves }\end{array}$ & $\begin{array}{l}\text { Leo en } \\
\text { forma } \\
\text { silenciosa. } \\
\text { Concentra- } \\
\text { ción }\end{array}$ \\
\hline Estudiante $\mathrm{N}^{\circ} 11$ & Sintetizar & $\begin{array}{l}\text { Busco ma- } \\
\text { terial afin }\end{array}$ & $\begin{array}{l}\text { Trazar } \\
\text { líneas y } \\
\text { letras }\end{array}$ & Leer & $\begin{array}{l}\text { Elaboro } \\
\text { esquemas o } \\
\text { resumen }\end{array}$ & $\begin{array}{l}\text { Busco } \\
\text { espacios } \\
\text { ordenados } \\
\text { e ilumina- } \\
\text { dos }\end{array}$ \\
\hline Estudiante $\mathrm{N}^{\circ} 12$ & Sintetizar & $\begin{array}{l}\text { Leo el } \\
\text { resumen } \\
\text { y trato de } \\
\text { entenderlo }\end{array}$ & $\begin{array}{l}\text { Trazar } \\
\text { líneas y } \\
\text { letras }\end{array}$ & Leer & $\begin{array}{l}\text { Subrayo } \\
\text { aspectos } \\
\text { importan- } \\
\text { tes. } \\
\text { Hago un } \\
\text { resumen de } \\
\text { lo subra- } \\
\text { yado }\end{array}$ & $\begin{array}{l}\text { Debe haber } \\
\text { completo } \\
\text { silencio. } \\
\text { Concentra- } \\
\text { ción }\end{array}$ \\
\hline Estudiante $\mathrm{N}^{\circ} 13$ & $\begin{array}{l}\text { Relacionar. } \\
\text { Compren- } \\
\text { der }\end{array}$ & Aprendo & & Leo & & $\begin{array}{l}\text { Escucho } \\
\text { (auditivo) } \\
\text { Imagino } \\
\text { (creativi- } \\
\text { dad) }\end{array}$ \\
\hline Estudiante $\mathrm{N}^{\circ} 14$ & $\begin{array}{l}\text { Analizar. } \\
\text { Compren- } \\
\text { der }\end{array}$ & & $\begin{array}{l}\text { Trazar } \\
\text { líneas y } \\
\text { letras }\end{array}$ & Leer & $\begin{array}{l}\text { Utilizo } \\
\text { mapa con- } \\
\text { ceptual. } \\
\text { Elaboro } \\
\text { esquemas }\end{array}$ & $\begin{array}{l}\text { Estudio en } \\
\text { un lugar } \\
\text { aislado. } \\
\text { Requiero } \\
\text { concentra- } \\
\text { ción }\end{array}$ \\
\hline Estudiante $\mathrm{N}^{\circ} 15$ & $\begin{array}{l}\text { Compren- } \\
\text { der }\end{array}$ & $\begin{array}{l}\text { Hago es- } \\
\text { fuerzo por } \\
\text { compren- } \\
\text { der. } \\
\text { Reflexionar }\end{array}$ & & & & $\begin{array}{l}\text { Pongo } \\
\text { atención } \\
\text { (auditivo). }\end{array}$ \\
\hline Estudiante $\mathrm{N}^{\circ} 16$ & Aplicar & & & Leer & $\begin{array}{l}\text { Aprendo } \\
\text { leyendo. } \\
\text { Explico y } \\
\text { ejemplifico }\end{array}$ & $\begin{array}{l}\text { Verbal- } \\
\text { lingüística }\end{array}$ \\
\hline Estudiante $\mathrm{N}^{\circ} 17$ & $\begin{array}{l}\text { Sintetizar. } \\
\text { Relacionar }\end{array}$ & & $\begin{array}{l}\text { Trazar } \\
\text { líneas }\end{array}$ & Leer & $\begin{array}{l}\text { Elaboro } \\
\text { mapa con- } \\
\text { ceptual. } \\
\text { Resumo } \\
\text { mental- } \\
\text { mente los } \\
\text { puntos } \\
\text { modulares }\end{array}$ & $\begin{array}{l}\text { Verbal-lin- } \\
\text { güística. } \\
\text { Abstrac- } \\
\text { ción }\end{array}$ \\
\hline
\end{tabular}




\begin{tabular}{|c|c|c|c|c|c|c|}
\hline Estudiante $\mathrm{N}^{\circ} 18$ & $\begin{array}{l}\text { Analizo, } \\
\text { estudio y } \\
\text { memorizo } \\
\text { la informa- } \\
\text { ción }\end{array}$ & $\begin{array}{l}\text { Pongo } \\
\text { atención }\end{array}$ & $\begin{array}{l}\text { Trazar } \\
\text { letras }\end{array}$ & Leer & $\begin{array}{l}\text { Escribo } \\
\text { la materia } \\
\text { para que se } \\
\text { me grabe }\end{array}$ & $\begin{array}{l}\text { Aprendo } \\
\text { de manera } \\
\text { visual y } \\
\text { auditiva. } \\
\text { Hago } \\
\text { mezcla } \\
\text { de los dos } \\
\text { sentidos }\end{array}$ \\
\hline Estudiante $\mathrm{N}^{\circ} 19$ & $\begin{array}{l}\text { Relacionar. } \\
\text { Asociar }\end{array}$ & $\begin{array}{l}\text { Estudio } \\
\text { sola en mi } \\
\text { casa y en la } \\
\text { noche. } \\
\text { Interiorizo } \\
\text { la materia }\end{array}$ & $\begin{array}{l}\text { Trazar } \\
\text { líneas }\end{array}$ & $\begin{array}{l}\text { Leer. } \\
\text { Escuchar. } \\
\text { Marcar }\end{array}$ & $\begin{array}{l}\text { Escucho } \\
\text { música. } \\
\text { Resalto con } \\
\text { un mar- } \\
\text { cador los } \\
\text { elementos } \\
\text { relevantes }\end{array}$ & $\begin{array}{l}\text { Concentra- } \\
\text { ción. } \\
\text { Auditiva }\end{array}$ \\
\hline Estudiante $\mathrm{N}^{\circ} 20$ & $\begin{array}{l}\text { Analizar. } \\
\text { Relacionar }\end{array}$ & $\begin{array}{l}\text { Los temas } \\
\text { abstractos } \\
\text { son indes- } \\
\text { cifrables }\end{array}$ & & Leer & $\begin{array}{l}\text { Aprendo } \\
\text { relacionan- } \\
\text { do }\end{array}$ & $\begin{array}{l}\text { Expe- } \\
\text { riencias } \\
\text { concretas }\end{array}$ \\
\hline
\end{tabular}

En la matriz anterior, se puede observar que sus respuestas hacen referencia a una serie de técnicas que implican todo un proceso desde los distintos niveles del dominio del conocimiento, los cuales requieren de ciertas actividades para alcanzar el aprendizaje de los estudiantes; tal es el caso de la lectura frecuente que se utiliza para el logro de un determinado objetivo.

Los datos muestran los diferentes estilos que los veinte estudiantes universitarios tienen para estudiar; se puede constatar que los niveles de aprendizaje mostrados son los que implican mayor complejidad en los procesos de aprendizaje (la reflexión, la comprensión, la explicación, la relación con objetos, el análisis, la síntesis). Estos procesos van acompañados de niveles básicos (el recordar o el uso de la memoria) y de técnicas que implican mayor abstracción y análisis en los procesos (el resumen, mapas conceptuales, esquemas y la observación).

Es importante destacar el uso de niveles afectivos o actitudinales, por ejemplo, el ponerse en actitud de aprendizaje, la disponibilidad para asimilar el conocimiento, la búsqueda de material, la investigación para ampliar conocimientos y poner en práctica lo aprendido.

Se reflejan en menor medida, los aspectos que tienen que ver con las destrezas o aptitudes, en este caso, algunos manipulan objetos como lápices y marcadores para subrayar o confeccionar mapas conceptuales o esquemas. 


\section{Consideraciones finales}

En este trabajo, se han enfocado dos perspectivas diferentes de abordar el aprendizaje de los estudiantes; una podría decirse que tiene un carácter más específico, porque está referida a los aspectos que se desarrollan en la práctica pedagógica; la otra tiene un carácter más social y se interesa por los aspectos extemos a la práctica pedagógica. En esta última, se determinan los problemas que afectan el aprendizaje de las y los estudiantes.

Por eso, Rafael Cuevas y Gerardo Morales afirman que los y las estudiantes, que ingresan a la universidad, ya traen un capital simbólico y heredado, porque el medio en el cual se han desarrollado los ha moldeado y más bien enfocan el problema de un "determinismo social", término usado por Bordieu.

Otros estudiosos, como Gardner, ven de forma distinta el problema del aprendizaje. Este afirma que es cierto que cada persona trae una inteligencia genética, pero que esta puede desarrollar su capacidad conforme sus experiencias, el medio que le rodea y la educación recibida.

En la consulta que se hizo a los veinte estudiantes universitarios, a la pregunta, ¿cómo aprende usted?, se observa en sus respuestas que existe mucha variedad de las formas en que estos aprenden; además, se refleja que las técnicas y los niveles de conocimiento para estudiar en cada caso son muy particulares, pues unos utilizan técnicas más complejas, con mayor capacidad de análisis. Podría pensarse que desde el punto de vista que se refieren Cuevas y Morales, los y las estudiantes han tenido espacios más reflexivos y críticos y una mejor formación académica, tal es el caso de quienes promueven un aprendizaje más eficaz mediante la investigación, la constante interrogación y la búsqueda de nueva información para complementar sus conocimientos; otros por el contrario, se quedan en las actividades y técnicas, pero no trascienden al conocimiento, posiblemente porque su papel como estudiantes ha sido muy pasivo.

Este estudio refleja un perfil del estudiante universitario que concibe el aprendizaje con estilo y capacidad diferentes y, desde luego, que estos resultados permiten a los docentes, la reflexión y el cuestionamiento de cómo enseñar en las aulas universitarias. 


\section{Referencias}

Binkley et al. (2004). Ayudando a los niños a triunfar en la escuela. Estilos de aprendizaje, de http://www.urbanext.uiuc.edu/succeed_sp/index.html.

Brockbank, A. (2002). Aprendizaje reflexivo en la educación superior. Capítulo III. Madrid: MORATA.

Cuevas Molina, Rafael. (2004). Tambor, de http://www.una.ac.cr.html.

Gardner, H. 2006. Inteligencias múltiples, de http://www.wikilearnig.com.wikilearning.com/gardner_inteligencias_multiples-wkc-12259.htm -

La Palma, F. (2004). Inteligencias múltiples, de http://www.aldeae.net/aldeae/aldea/ tareas2.

Morales, G. (2004). En http://www.una.ac.cr. www.fcs.una.ac.cr/fcs/documentos/ sociedad\%20posible\%20en\%20America\%20Latina.pdf.

Nowalski, J. (2003). Asimetrias económicas, sociales y politicas en Costa Rica: hacia una calidad de vida digna. San José, Costa Rica.

Ortiz, Terry. (2004). Los maestros asumen un nuevo papel en el salón de clase, de http://www.sedl.org/pubs/family91/3a.html.

Solano, A. (2004). En: Opiniones Tambor. www.elacvirtual.net/documents/conferencias elac/III_conferencia/libro intro.

Valverde, H. (2004). En: Tambor Opiniones, de http://www.una.ac.cr. 